Background Retturn to play decisions are complex. Pathways considering effective communication should be considered. High correlations between sports medicine/management communication and athlete injuries have been reported.

Objective Design and implement a pratical sports medicine/ management communication tool with UCD Athletic Football Club's (AFC) Premier division team for an 8 week trial.

Design Prospective Exploratory study.

Setting UCD Physiotherpy Hub- physiotherapists, supported by students provide sports physiotherapy services. UCD AFC's top tier team competes in the Irish Premier Division.

Patients (or Participants) UCD AFC team selected through puposive sampling. Coaches approached seeking qualitative feedback regarding the communication tool. Only de-identified data reported.

Main Outcome Measurements 'Traffic Light System': Model chosen based on sports medicine and management staff consensus. Players were categorised as Green-fit to play, Yellow-fit to play with caution, Amber-fit for light training only or Rednot fit to play. Classification was based on combining return to play criteria/outcome measures and clinical decision making by the Physiotherapist. Player availability (group and individual) in pie chart and table format was shared with management prior to training sessions/games.

Qualitative Feedback Management feedback gathered through a survey at the end of the 8 week trial.

Results Descriptive results Twenty seven UCD Soccer athletes of mean age $20.26 \pm 1.3$ years monitoried over an 8 week mid-season period. Sixteen initial Physiotherapy clinical sessions completed. Average availability 69\% Green 8.6\% Yellow 9.3\% Amber 13\% Red.

Qualitative results Surveys were completed by 3 AFC Management staff. Key themes: i) Ease of interpretation ii) Application to training session/game planning iii) Improving availability and transparency

Conclusions The 'Traffic light System' was time efficient, low cost, and has received positive preliminary feedback. Trialling the system over a full season is needed to guage long term utility.

\section{THE BAN OF TRAMADOL AND HAZARD PREVENTION IN CYCLING}

${ }^{1}$ Xavier Bigard, ${ }^{3}$ Angeline Turin, ${ }^{2}$ Olivier Salamin, ${ }^{2}$ Raphael Faiss, ${ }^{5}$ Youssef Daali, ${ }^{4}$ Serge Rudaz, ${ }^{3}$ Francesca Rossi, ${ }^{2}$ Martial Saugy. ${ }^{1}$ Union Cycliste Internationale, Aigle, Switzerland; ${ }^{2}$ Center of Research and Expertise in anti-Doping sciences, Lausanne, Switzerland; ${ }^{3}$ Cycling Anti-Doping Foundation, Aigle, Switzerland; ${ }^{4}$ Analytical Sciences School of Pharmaceutical Sciences, Geneva, Switzerland; ${ }^{5}$ Centre Médical Universitaire, Geneva, Switzerland

\subsection{6/bjsports-2021-IOC.334}

Background Tramadol is a synthetic opioid not banned by WADA, but included in the Monitoring Program since 2012. The prevalence of use of tramadol in competition was approximately $5 \%$ in cycling. These data clearly suggest that there was a misuse of tramadol in cycling. Opioids have several adverse effects, including nausea, dizziness and reduced vigilance. Such effects might be a potential cause of falls during races. Moreover, the psychoactive effects of tramadol are known to lead to abuse and then to drug dependence.

Objective In order to decrease the risk of falls and prevent the occurrence of opioid-related side effects, the UCI has decided to ban the use of tramadol in competition from the $1^{\text {st }}$ March 2019. This regulation was part of a vast program of injury prevention in cycling, and was included in the UCI medical rules.

Main Outcome Measurements The tramadol controls are done in dried blood spots (DBS). The capillary blood is taken from a finger using a specific device that allows reliable collection (Hemaxis-DB10, DBS-System SA, Gland, Switzerland). DBS samples are sent to the reference laboratory in sealed bags through a reliable courier company. Tramadol analyses are performed by using the UHPLC-MS/MS technique. Positive results are based on the presence of the parent compound and its two main metabolites. The results are reviewed by an independent third entity, and sent to the UCI Medical Director. Positive controls are sanctioned with disqualification of the event and a fine.

Results As this summary is being written, 520 controls have been done in UCI-WorldTour races and World Championships. No positive results were reported.

Conclusions The ban on tramadol in cycling is a UCI medical regulation totally independent of the WADA rules, and supported by medical reasons intended to prevent the risks of injuries and opioid-related side effects. The introduction of the Tramadol-DBS tests has obviously a strong deterrent effect on its use in cycling.

\section{INJURIES AMONG YOUTH MOUNTAIN BIKE RACERS: THREE-YEAR DATA FROM A NATION-WIDE INJURY SURVEILLANCE SYSTEM IN THE UNITED STATES}

${ }^{1}$ Meredith Ehn, 'Daniel M Cushman, ${ }^{1}$ Masaru Teramoto, ${ }^{2}$ Chris Spencer, 'Stuart Willick. ${ }^{1}$ University of Utah, Salt Lake City, USA; ${ }^{2}$ National Interscholastic Cycling Association, Berkeley, USA

\subsection{6/bjsports-2021-IOC.335}

Background Youth mountain bike racing is a rapidly growing sport in the United States. An injury surveillance system (ISS) is now implemented through a collaboration between University of Utah researchers and the National Interscholastic Cycling Association (NICA), referred to as NICA-ISS, to better understand injury characteristics in this sport and pursue datadriven injury prevention strategies.

Objective Describe types of and factors associated with injuries sustained by youth mountain bike racers during NICA-sanctioned mountain bike racing and training.

Design NICA-ISS was developed using a web-based system. Data were collected from NICA leagues during the 20182020 seasons. Designated reporters on each team were asked to complete weekly incident and exposure report forms.

Setting Nation-wide youth mountain biking leagues in the United States.

Participants Student-athletes on NICA teams in grades 8-12 participating in the 2018-2020 seasons.

Assessment of Risk Factors Variables analyzed included injury characteristics, athlete demographics, trail conditions, incline during crash, weather, crash circumstances (practice, race, passing, trail familiarity), and health factors (dehydration, sleep, illness).

Main Outcome Measurements Proportions/rates of injuries overall, by type and location, and by group of athletes.

Results Injuries were tracked in 66,588 student-athlete-years, resulting in 2,587 injuries in 1,677 student-athlete injury events reported. The most commonly reported injury was 